\title{
Preparation and low temperature stability of PbTe nanocrystals embedded in ZnTe matrix by hot-wall deposition
}

\author{
Seishi Abe ${ }^{1}$
}

Received: 13 January 2016/Accepted: 16 April 2016/Published online: 28 April 2016

(c) The Author(s) 2016. This article is published with open access at Springerlink.com

\begin{abstract}
Preparation of PbTe-ZnTe nanocomposite thin film is investigated by one-step synthesis using hot-wall deposition with multiple solid-state resources of $\mathrm{PbTe}$ and ZnTe. X-ray diffraction and Raman spectrum indicate that composite thin films contain $\mathrm{PbTe}$ with $\mathrm{NaCl}$ structure, and ZnTe with zinc-blende structure. High-resolution transmission electron microscopy and fast Fourier transform analysis indicate that isolated $\mathrm{PbTe}$ nanocrystalline facets are dispersed in ZnTe matrix. The two compounds phaseseparate at hetero-interface without forming a ternary solid solution $\mathrm{Zn}_{1-x} \mathrm{~Pb}_{x} \mathrm{Te}$. Therefore, simultaneous evaporation of the multiple resources provides a phase-separating nanocomposite with faceted PbTe nanocrystals embedded in $\mathrm{ZnTe}$ matrix. The $\mathrm{PbTe}$ crystallizes even at a relatively low substrate temperature of $274 \mathrm{~K}$.
\end{abstract}

Keywords One-step physical synthesis - Nanocomposite thin film · Physical vapor deposition - Simultaneous evaporation · Insoluble material system · High-resolution transmission electron microscopy

\section{Introduction}

Nanoscale semiconductor embedded in a wide-gap material has potential application for quantum dot solar cells due to tunableness for absorbing effective energy region in solar radiation spectrum and impact ionization for producing multiple electron-hole pairs per photon [1].

Seishi Abe

abe@denjiken.ne.jp

1 Research Institute for Electromagnetic Materials, 2-1-1 Yagiyama-minami, Sendai 982-0807, Japan
Nanocomposites with different combinations in nanocrystal and matrix were proposed using chemical or physical preparation techniques [2-9].

A combination of lead and zinc chalcogenide system is valuable for preparing a nanocomposite thin film due to the reasons as follows: (1) this system phase-separates in thermal equilibrium without forming a solid solution, (2) band-gap difference between lead and zinc chalcogenide is relatively large, capable of exhibiting quantum confinement effects, and (3) vapor pressures of lead and zinc chalcogenide are similar and relatively large, capable of simultaneously thermal-evaporating the solid-state substances. Specifically, a phase separation provides a compositional steepness at a hetero-interface between lead and zinc chalcogenide with a relatively large band-gap difference. To retain the most important characteristic of phase separation between lead and zinc chalcogenide, a technique of hot-wall deposition (HWD) should be employed due to a state near thermal equilibrium in a deposition atmosphere [10]. Based on these material designs, we proposed a composite thin film with $\mathrm{PbSe}$ nanocrystals embedded in a ZnSe matrix by one-step synthesis using HWD with multiple evaporation sources of $\mathrm{PbSe}$ and $\mathrm{ZnSe}$ [11]. Furthermore, this material design with theuse of HWD exhibits a unique characteristic of vapor-phase reaction. Evaporation sources of $\mathrm{PbTe}$ and $\mathrm{ZnSe}$ produce a nanocomposite containing $\mathrm{ZnSe}$ and nanocrystalline $\mathrm{PbSe}$ with slight inclusion of $\mathrm{Te}(\mathrm{PbSe}: \mathrm{Te})$, transforming from $\mathrm{PbTe}$ (evaporation source) to PbSe:Te (film production) [12]. Different evaporation sources ( $\mathrm{PbSe}$ or $\mathrm{PbTe})$, therefore, provide similar nanocrystalline $\mathrm{PbSe}$, but the XRD peaks of pure $\mathrm{PbSe}$ nanocrystals are weak even at a relatively high substrate temperature of $403 \mathrm{~K}$ [13], whereas nanoscale PbSe:Te crystallizes even at $274 \mathrm{~K}$ [12]. These results suggest that slight inclusion of $\mathrm{Te}$ in $\mathrm{PbSe}$ nanocrystal 
stabilizes the crystalline phase. Further investigation is needed whether pure PbTe nanocrystal actually stabilizes at a relatively low temperature. Moreover, a composite with $\mathrm{PbSe}$ nanocrystals embedded in $\mathrm{ZnSe}$ matrix exhibited n-type conductivity in our preliminary experiment, while compound semiconductor ZnTe tends to exhibit p-type conductivity [14]. Hence, composites with $\mathrm{Pb}$ and Zn chalcogenide system will possibly provide a $\mathrm{p}-\mathrm{n}$ junction. Conductivity type should be investigated on ZnTe prepared by the HWD thus employed. Therefore, the present study employs a nanocomposite thin film with PbTeZnTe system. This system phase-separates at temperatures below $1160 \mathrm{~K}$ in bulk phase diagram [15]. Also, a nanocomposite thin film of PbTe nanocrystal embedded in a ZnTe matrix is capable of exhibiting quantum size effects because of the relatively large exciton Bohr radius of $46 \mathrm{~nm}$ in PbTe [16], and the relatively large band-gap difference between ZnTe (2.25 eV) [17] and PbTe $(0.31 \mathrm{eV})$ [18] with type-I band offset [19]. Also, vapor pressure of PbTe [20] is similar to that of ZnTe [21]. Hence, simultaneous evaporation of solid-state sources (PbTe and $\mathrm{ZnTe}$ ) is available for preparing a nanocomposite thin film with PbTe nanocrystals embedded in $\mathrm{ZnTe}$ matrix using HWD.

\section{Experimental}

A nanocomposite thin film was prepared using HWD described elsewhere [11]. Here, PbTe and ZnTe were used as evaporation sources with $5 \mathrm{~N}$ purity (Furuuchi Chemical, Tokyo, Japan). The two sources were located at different positions in a quartz liner to simultaneously thermal evaporate on a glass (Corning Eagle 2000, NY, USA) or Si substrate. The substrate temperature was changed by circulating water controlled at temperatures from 274 to $350 \mathrm{~K}$. The temperatures were kept constant at $843 \mathrm{~K}$ for a source of ZnTe, and $683 \mathrm{~K}$ for the wall, which is located at another position without any source in the quartz liner. The temperature for a source of PbTe was varied from 713 to $778 \mathrm{~K}$, to provide different PbTe concentrations. The film was structurally characterized using X-ray diffraction (XRD) with $\mathrm{Cu} \mathrm{K} \alpha$ radiation (Rigaku RAD-X, Tokyo, Japan). A symmetric $2 \theta-\theta$ configuration was used. The composition of the film was analyzed using energy dispersive spectroscopy (EDS) (EDAX model, Phoenix, NJ, USA) operating at $10 \mathrm{kV}$ with standard samples of PbTe to calibrate the analyzed results for $\mathrm{Pb}$ and $\mathrm{Te}$, and with $\mathrm{ZnSe}$ for $\mathrm{Zn}$. The Raman spectrum of the film was measured using a Raman spectrometer (JASCO NRS-5000, Tokyo, Japan) with a pumping semiconductor laser at $532 \mathrm{~nm}$. The film was directly observed using transmission electron microscopy (TEM) operating at $300 \mathrm{kV}$ (Hitachi
H-9000NAR, Tokyo, Japan). Ion milling was performed in the sample preparation. Current-voltage curve and photocurrent spectrum of the film were measured using photocurrent spectrometer (BUNKOUKEIKI, Tokyo, Japan).

\section{Results and discussion}

Figure 1 depicts the XRD pattern of the thin films as a function of the concentration of $\mathrm{Pb}$. In this case, the substrate temperature is kept constant at $350 \mathrm{~K}$. Simultaneous evaporation enables multiple inclusions of elements $(\mathrm{Pb}$, $\mathrm{Zn}$, and Te), due to similar vapor pressures in PbTe [20] $\left[\log (p / \mathrm{atm})=-11636 T^{-1}+7.946\right.$, where $T$ is Kelvin temperature] and $\mathrm{ZnTe}[21]\left[\log (p / \mathrm{atm})=-10627 T^{-1}+\right.$ 6.771]. The concentration of $\mathrm{Pb}$ can be controlled by the source temperature of PbTe. In the figure, a zinc-blende structure is first observed at 0.7 at. $\% \mathrm{~Pb}$ with dominantly [111] orientation despite the use of a glass substrate. A different phase with $\mathrm{NaCl}$ structure appears at 0.8 at. $\% \mathrm{~Pb}$ and any peak attributing to elements ( $\mathrm{Zn}, \mathrm{Pb}$, and $\mathrm{Te}$ ) cannot be seen in the pattern. Hence, the polycrystalline films form a phase mixture of zinc-blende structure and $\mathrm{NaCl}$ structure. The composition ratio in cation with $\mathrm{Zn}$ and $\mathrm{Pb}$, and anion with Te tends to deviate Te-excess from stoichiometry in almost samples. In thermal evaporation of sources (PbTe and ZnTe), PbTe sublimates as a form of binary compound with species of $98 \%$ in vapor phase [22] $[\operatorname{PbTe}(\mathrm{s})=\operatorname{PbTe}(\mathrm{g})]$, while ZnTe dissociates to each element [23] $\left[\mathrm{ZnTe}(\mathrm{s})=\mathrm{Zn}(\mathrm{g})+1 / 2 \mathrm{Te}_{2}(\mathrm{~g})\right]$. Hence, a deviation from stoichiometry dominantly depends on the dissociated elements ( $\mathrm{Zn}$ and Te). The Te-excess tendency suggests that the condensation coefficient of Te exceeds that of $\mathrm{Zn}$.

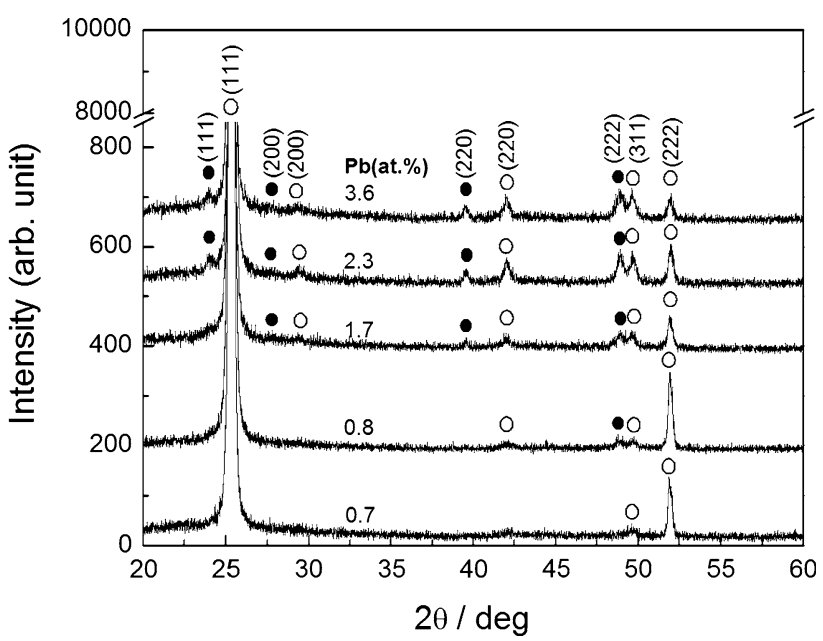

Fig. 1 XRD pattern of thin films with respect to the source temperature of $\mathrm{PbTe}$. Black dots indicate a phase with $\mathrm{NaCl}$ structure and circles indicate a phase with zinc-blende structure 
Figure 2a depicts the lattice constant of the thin films as a function of the concentration of $\mathrm{Pb}$. The lattice constant is estimated from the XRD peak at (111) Bragg reflection for zinc-blende structure and the peak at $(220)$ for $\mathrm{NaCl}$ structure. The dotted lines indicate the lattice constants of standard $\mathrm{ZnTe}$ and $\mathrm{PbTe}$. The lattice constant of the $\mathrm{NaCl}$ structure is almost the same to that of the standard $\mathrm{PbTe}$ regardless of the concentration of $\mathrm{Pb}$. If a solid solution is formed in $\mathrm{PbTe}$ with element $\mathrm{Zn}$, the lattice constant should decrease with increasing concentration of $\mathrm{Pb}$, due to the difference in radii between $\mathrm{Pb}$ and $\mathrm{Zn}$. Therefore, the phase with $\mathrm{NaCl}$ structure is reasonably assigned to $\mathrm{PbTe}$. In the phase with zinc-blende structure, the lattice constant is slightly smaller than that of standard $\mathrm{ZnTe}$ and is almost unchanged regardless of the concentration of $\mathrm{Pb}$. Figure $2 \mathrm{~b}$ indicates the optical absorption spectrum of the thin film with 0.7 at. $\% \mathrm{~Pb}, 47.8$ at. $\% \mathrm{Zn}$, and 51.5 at. $\% \mathrm{Te}$. The spectrum exhibits an optical absorption edge with an energy band gap of $2.24 \mathrm{eV}$, corresponding well to that of $\mathrm{ZnTe}(2.25 \mathrm{eV})$ [17]. Furthermore, Raman spectrum of the film (Fig. 2c) contains four peaks at $206 \mathrm{~cm}^{-1}$ and its phonon replica is in good agreement with the Raman spectrum of $\mathrm{ZnTe}$ due to a longitudinal optical phonon mode (1LO) at $206.5 \mathrm{~cm}^{-1}$ [24]. Hence, the phase with zinc-blende structure is reasonably assigned to $\mathrm{ZnTe}$ without alloying with $\mathrm{Pb}$. Therefore, the thin films form a phase mixture of $\mathrm{PbTe}$ with $\mathrm{NaCl}$ structure and $\mathrm{ZnTe}$ with zinc-blende structure. The two compounds (PbTe and $\mathrm{ZnTe})$ are therefore found to phase-separate even in the film production following the bulk phase diagram [15], during a simultaneous evaporation of solid-state sources ( $\mathrm{PbTe}$ and $\mathrm{ZnTe}$ ). This result also indicates that a deposition process using HWD provides a state with thermal equilibrium, since a preparation technique such as molecular beam epitaxy with thermally non-equilibrium provides a solid solution with a solubility range beyond that at thermal equilibrium [25].

Figure 3a presents a TEM plane view image of the thin film with 3.6 at. $\% \mathrm{~Pb}, 44.9$ at. $\% \mathrm{Zn}$, and 51.5 at. $\% \mathrm{Te}$ (i.e., about 7 at.\%PbTe). The morphology is found to form a nanocomposite with slightly dark isolated nanoparticles dispersed in a bright matrix. The nanoparticles are well developed with sizes of 30-100 nm. In particular, it should be noted that faceted nanoparticles are frequently observed. The inset in Fig. 3a presents an enlarged image of typical faceted nanoparticle as denoted by the dotted line in Fig. 3a. A hetero-interface between the faceted nanocrystal and a crystalline matrix can be observed. The composite thin film forms polycrystalline structure (Fig. 1), but the geometric shape of the facet enables a fine observation of a lattice alignment at a nanoscale hetero-interface. Figure $3 \mathrm{~b}$ represents high-resolution TEM image of the dotted square area in the inset in Fig. 3a. It is clearly found that the lattice fringes with plane widths of $0.228 \mathrm{~nm}$ at $(0-22)$ in $\mathrm{PbTe}$ and $0.352 \mathrm{~nm}$ at (111) in ZnTe are perpendicularly aligned at the hetero-interface. The clear hetero-interface indicates that the two compounds phase-separate without forming an intermediate phase such as ternary solid solution $\mathrm{Zn}_{1-x}$ $\mathrm{Pb}_{x} \mathrm{Te}$. The strict change from $\mathrm{PbTe}$ to $\mathrm{ZnTe}$ at the heterointerface (Fig. 3b) is expected to produce a steep energy potential barrier in $\mathrm{PbTe}$ nanocrystal for effectively confining an electron excited by absorbing light. Fast Fourier transform (FFT) analysis provides the details of the local alignment in the faceted nanoparticle. Figure $3 \mathrm{c}$, d depicts the corresponding FFT diffraction pattern of the faceted nanocrystal and the matrix in Fig. 3b. The diffraction pattern can be indexed to cubic PbTe and ZnTe. In Fig. 3c, the diffractions with label- $\mathrm{A}, \mathrm{B}$, and $\mathrm{C}$ are assigned to
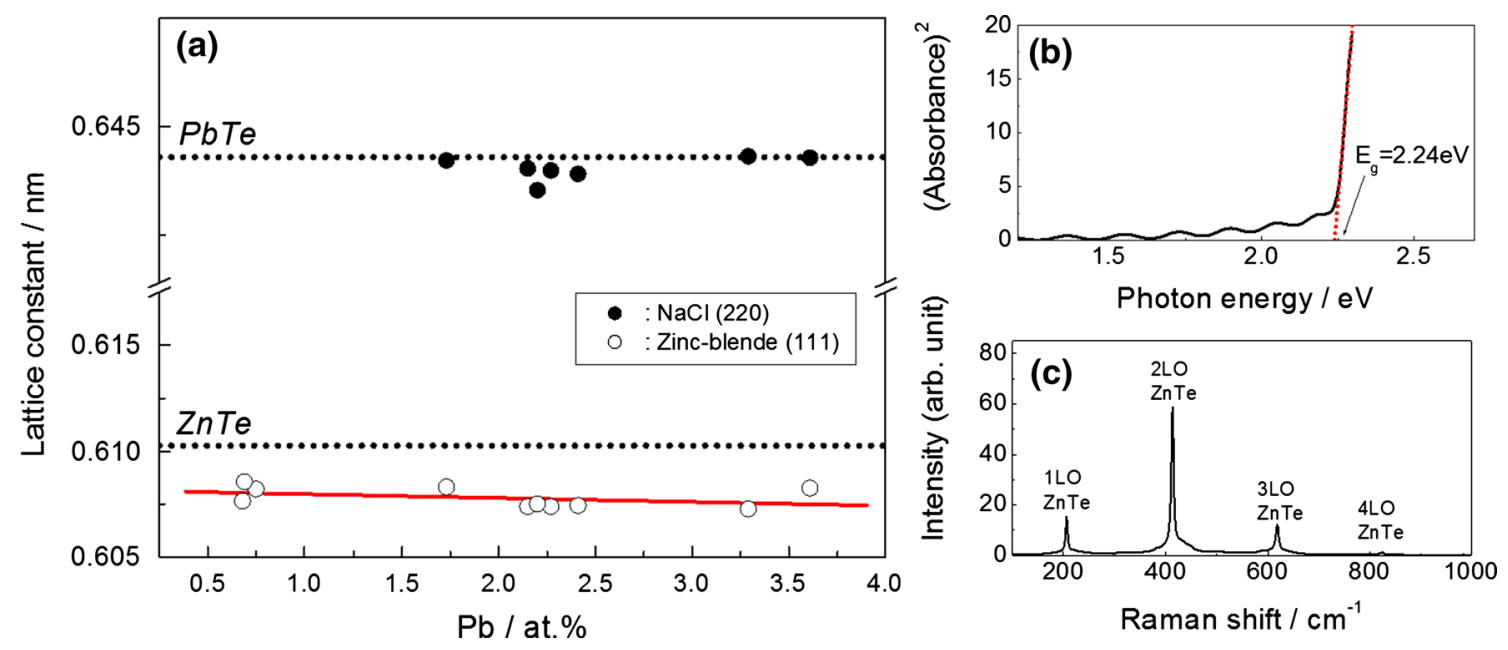

Fig. 2 a Lattice constant of thin films as a function of the source temperature of PbTe. b Optical absorption spectrum and $\mathbf{c}$ Raman spectrum of a thin film with 0.7 at. $\% \mathrm{~Pb}$ 
Fig. 3 Direct observation of a thin film with 3.6 at. $\% \mathrm{~Pb}$. a Plane view TEM image. Inset indicates an enlarged image of the dotted area. b Highresolution TEM image of the selected area denoted by square in the inset in a. c FFT diffraction pattern of the slightly dark nanocrystalline facet in b. d FFT diffraction pattern of the bright area in $\mathbf{b}$

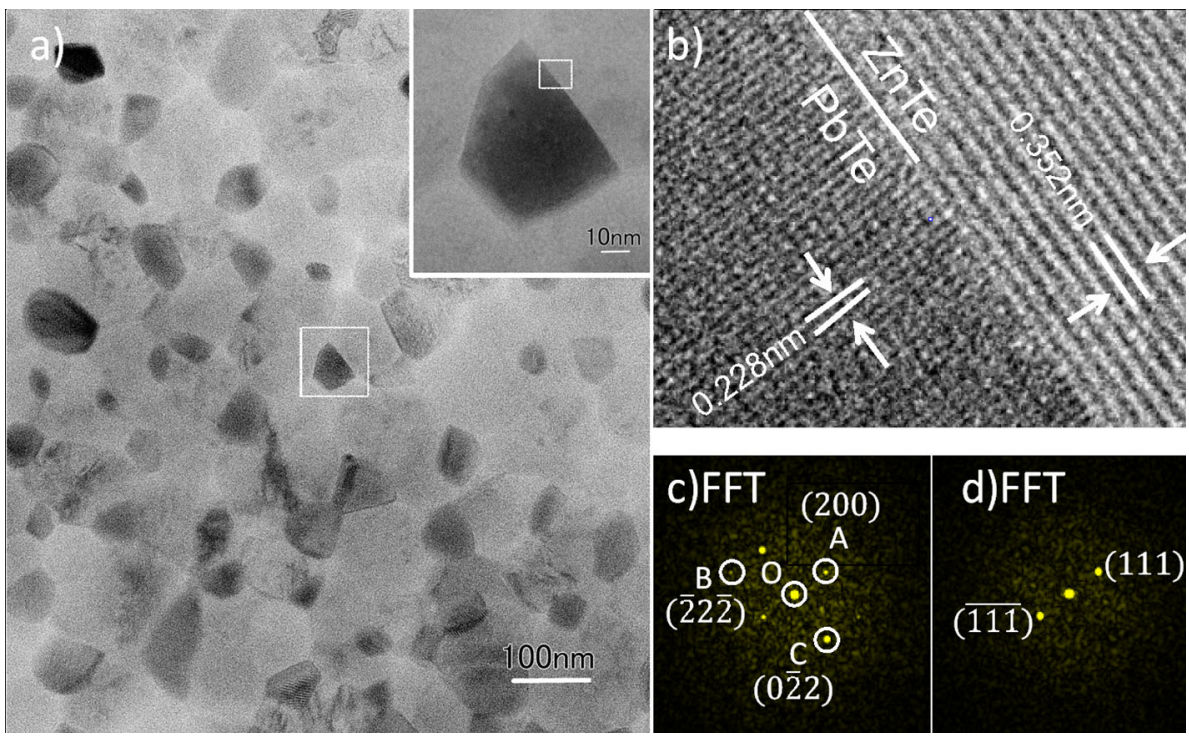

cubic $\mathrm{PbTe}$ with a plane width of $0.326 \mathrm{~nm}$ at (200), $0.185 \mathrm{~nm}$ at $(-22-2)$, and $0.228 \mathrm{~nm}$ at $(0-22)$. The angles A-O-B, A-O-C, and B-O-C are $126^{\circ}, 89^{\circ}$, and $145^{\circ}$, respectively. The lattice alignment of the nanocrystal corresponds to that of the standard data (JCPDS 38-1435), observing along the [011] direction. In Fig. 3d, the spots are assigned to the cubic $\mathrm{ZnTe}$ with a plane width of $0.352 \mathrm{~nm}$ at (111) and $(-1-1-1)$, corresponding well to the standard data in cubic ZnTe (JCPDS 15-0746). The results indicate that a growth direction of [111] in $\mathrm{ZnTe}$ aligns along that of [100] in PbTe. Furthermore, additional FFT-analysis (not shown here) also indicates [111] orientation in ZnTe matrix even at a different hetero-interface of [111] in the PbTe facet. Also, a pure ZnTe polycrystalline film exhibits a dominant growth orientation of [111] despite the use of a glass substrate in our preliminary experiment. Therefore, the ZnTe matrix at the hetero-interface (Fig. 3b) is reasonably considered to be an adhesive growth on the PbTe facet. It is considered as a kinetics of producing such alignment that a faceted PbTe nanocrystal is first developed on a film surface, and vaporized elements ( $\mathrm{Zn}$ and $\mathrm{Te}$ ) are subsequently deposited on the faceted $\mathrm{PbTe}$ [e.g., at (200)] for constructing a ZnTe compound (i.e., the facet just plays a role like a nanoscale substrate), since the solid-state sources dominantly evaporate as a form of binary compound in $\mathrm{PbTe}$ [22] and dissociate to each element in $\mathrm{ZnTe}$ [23]. The Te-excess tendency (Fig. 1) suggests that an element Te [i.e., (111)B] tends to first deposited on the $\mathrm{PbTe}$ facet prior to an element $\mathrm{Zn}$ [i.e., (111)A]. The faceted PbTe (inset in Fig. 3a) forms polyhedron shape. A nanocrystalline shape depends on a ratio of growth rate in [100] direction to that in [111] direction, forming cubic shape with a ratio of 0.58 , and octahedron shape with a ratio of 1.73 [26]. The size of
$\{200\}$ facet in the inset in Fig. 3a exceeds that of $\{-22-2\}$ facet. This result suggests that the ratio of growth rate in the faceted $\mathrm{PbTe}$ nanocrystal is less than 1 during the simultaneous evaporation with $\mathrm{PbTe}$ and $\mathrm{ZnTe}$. A ratio of growth rate in $\mathrm{ZnTe}$ to that in $\mathrm{PbTe}$ will also affect the shape of faceted PbTe nanocrystal, while the present study employs relatively low inclusion (i.e., low growth rate) of $\mathrm{PbTe}$ (Fig. 1). It is, therefore, found that one-step synthesis provides a nanocomposite thin film with faceted $\mathrm{PbTe}$ nanocrystals embedded in ZnTe matrix, with clear phase separation at hetero-interface.

In our previous study, a composite with $\mathrm{PbSe}$ nanocrystals embedded in $\mathrm{ZnSe}$ matrix exhibited n-type conductivity, whereas a compound semiconductor $\mathrm{ZnTe}$ tends to exhibit p-type conductivity [24]. Hence, composites with $\mathrm{Pb}$ and $\mathrm{Zn}$ chalcogenide system will possibly provide a $\mathrm{p}-\mathrm{n}$ junction. Conductivity type is next investigated on $\mathrm{ZnTe}$ employed as the matrix in the nanocomposite thin film. Figure 4a depicts current-voltage curves of ZnTe thin films prepared on Si substrate with n-type and p-type conductivity using HWD with an evaporation source of $\mathrm{ZnTe}$ without PbTe. ITO electrode with $20 \mathrm{~nm}$-thick is deposited on the ZnTe thin films and In-electrode is contacted to $\mathrm{Si}$ substrate as indicated in the inset. The $\mathrm{ZnTe}$ thin film on p-type $\mathrm{Si}$ exhibits rectifying behavior in the dark and photovoltaic characteristic with an open circuit voltage of $0.12 \mathrm{~V}$ under irradiation of solar simulator (AM1.5), whereas the ZnTe thin film on n-type Si provides a weak behavior in the dark. This result suggests that the $\mathrm{ZnTe}$ thin film has n-type conductivity. Hence, p-type $\mathrm{Si}$ is employed as a substrate for preparing the nanocomposite thin films to investigate photocurrent spectrum. Figure $4 \mathrm{~b}$ depicts typical normalized photocurrent spectra of the nanocomposite thin films prepared at a substrate 

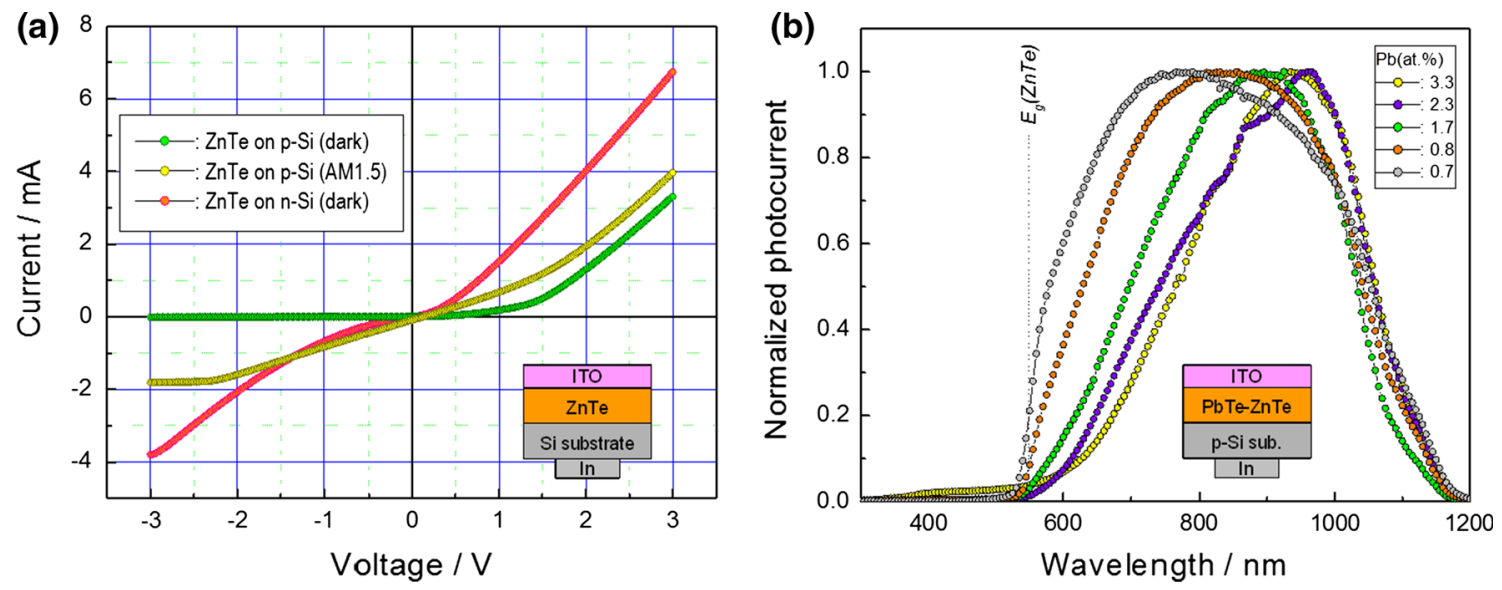

Fig. 4 a Current-voltage curve of ZnTe thin films and b normalized photocurrent spectrum of nanocomposite thin films

temperature of $350 \mathrm{~K}$. The broad spectra can be observed in the wavelength range. The photocurrent generates at wavelengths exceeding $549 \mathrm{~nm}$ as indicated by dotted line (energy band gap of $\mathrm{ZnTe}$ ) and wavelengths below $1200 \mathrm{~nm}$ that is considerably apart from the energy band gap of $\mathrm{PbTe}(4000 \mathrm{~nm})$. The PbTe nanocrystals exhibit no obvious quantum size effects due to their relatively large size (Fig. 3). Hence, the photocurrent reasonably depends on Si substrate. The normalized photocurrent decreases in visible wavelength range with increasing $\mathrm{Pb}$ concentration. This result indicates that an increase of PbTe nanocrystals with relatively large size in the nanocomposite reduces a photoresponse in visible range. Doping impurity will be needed to prepare p-type ZnTe.

Next, temperature dependence of substrate is investigated to clarify temperature stability of PbTe nanocrystals embedded in ZnTe matrix. Figure 5a depicts the XRD pattern of nanocomposite thin films with respect to substrate temperature. In this case, $\mathrm{Pb}$ concentration is kept constant at $5.3 \pm 0.5$ at.\% (except the sample at $274 \mathrm{~K}$ with 1.4 at. $\% \mathrm{~Pb}$ ), since mean grain size of PbTe minimizes at $5.3 \mathrm{at} \% \mathrm{~Pb}$ with respect to $\mathrm{Pb}$ concentration in the range from 1.7 to 6.1 at.\% in our preliminary experiment. Hence, most of the nanocomposite thin films are expected to contain about 10 at.\%PbTe. The dotted line indicates the peak position of standard $\mathrm{PbTe}$ and $\mathrm{ZnTe}$. The diffraction peaks in $\mathrm{PbTe}$ with $\mathrm{NaCl}$ structure and $\mathrm{ZnTe}$ with zincblende structure are clearly observed at $274 \mathrm{~K}$. Hence, the composite film with $\mathrm{PbTe}$ nanocrystals embedded in $\mathrm{ZnTe}$ matrix is crystallized even at a relatively low temperature of $274 \mathrm{~K}$. In our previous study, the XRD peak of PbSe in the $\mathrm{PbSe}-\mathrm{ZnSe}$ composite thin films is weak even at the relatively high temperature of $403 \mathrm{~K}$ [13]. Furthermore, nanoscale $\mathrm{PbTeSe}$ ternary solid solution crystallizes at a substrate temperature of $274 \mathrm{~K}$ in a composite film with ZnSe matrix [12]. The present result (Fig. 5a) strongly suggests that an inclusion of $\mathrm{Te}$ in $\mathrm{PbSe}$ stabilizes a nanocrystalline state embedded in $\mathrm{ZnSe}$ matrix at relatively low temperature. The mean grain size of $\mathrm{PbTe}$ is depicted in Fig. 5b. The mean grain size is estimated from the full width at half maximum of the diffraction peak, according to Scherrer's formula [27]. The size varies from 22 to $13 \mathrm{~nm}$ with respect to the substrate temperature, but there is no obvious tendency. It is, therefore, found in the $\mathrm{PbTe}-$ $\mathrm{ZnTe}$ nanocomposite thin films that both the compounds crystallize even at a substrate temperature of room temperature without any post-annealing.

One-step synthesis provides a nanocomposite thin film with faceted PbTe nanocrystals embedded in ZnTe matrix during simultaneous evaporation of solid sources $(\mathrm{PbTe}$ and $\mathrm{ZnTe}$ ) with similar vapor pressures. The composite has a strict hetero-interface between $\mathrm{PbTe}$ and $\mathrm{ZnTe}$, without forming a ternary solid solution such as $\mathrm{Zn}_{1-x} \mathrm{~Pb}_{x} \mathrm{Te}$. Hence, $\mathrm{PbTe}$ nanocrystal is expected to form a steep energy potential barrier with type-I band offset [19]. According to Brus model [28], the ground state transition of electron-hole pairs in PbTe nanocrystal is calculated to be $0.38 \mathrm{eV}$ with a mean grain size of $13 \mathrm{~nm}$ at $290 \mathrm{~K}$, which is the smallest size thus obtained in the present study. The estimated band gap is close to that of bulk $\mathrm{PbTe}$ $(0.31 \mathrm{eV})$. The nanocomposite thin film actually exhibits broad optical absorption toward infrared range. This broad absorption suggests that quantum size effects are weak in the PbTe nanocrystals. Further decrease in grain size of $\mathrm{PbTe}$ nanocrystals is therefore needed to enhance quantum size effects.

\section{Conclusion}

We investigated one-step synthesis of a nanocomposite thin film with faceted $\mathrm{PbTe}$ nanocrystals embedded in $\mathrm{ZnTe}$ matrix using HWD with solid-state sources (PbTe and $\mathrm{ZnTe}$ ). Similar vapor pressures in the two compounds 

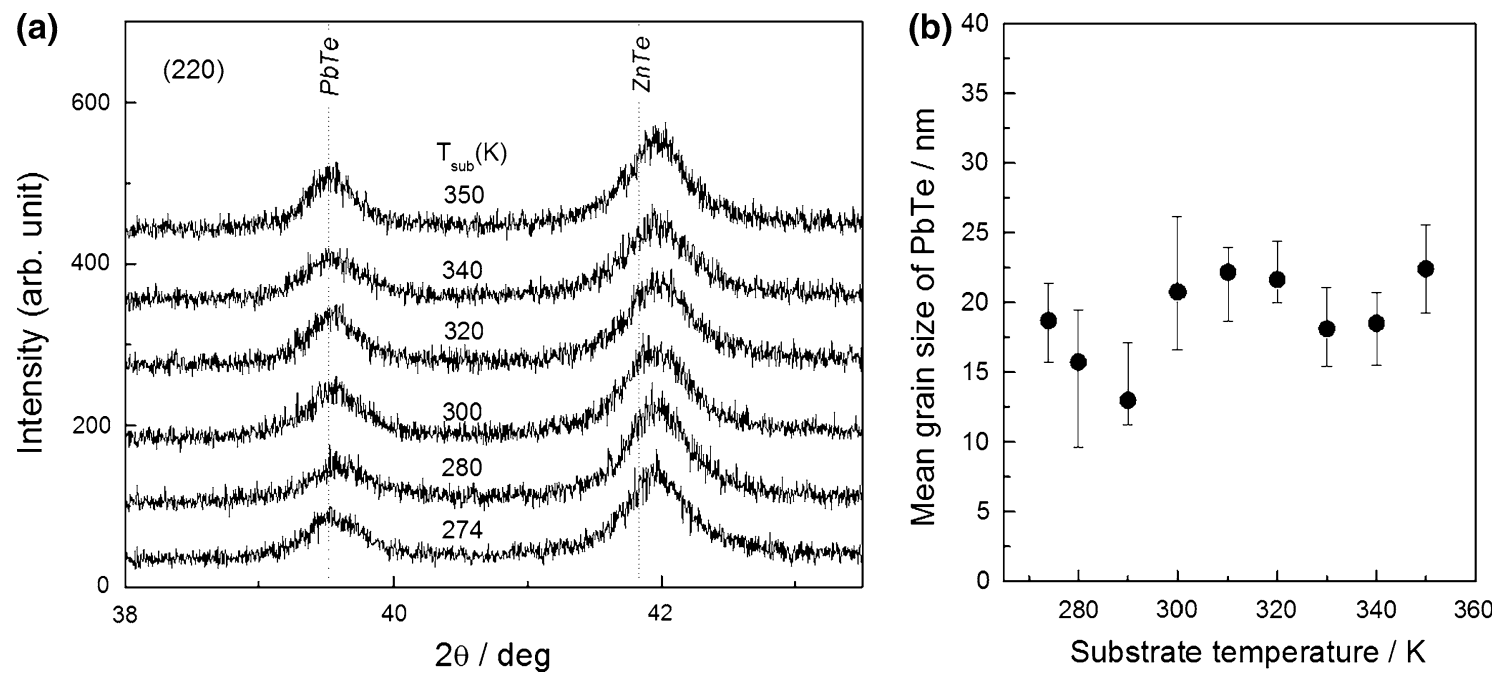

Fig. 5 XRD pattern of nanocomposite thin films with respect to substrate temperature. a $2 \theta$ range from $38^{\circ}$ to $43.5^{\circ}$. The dotted lines indicate the peak positions of standard $\mathrm{PbTe}$ and $\mathrm{ZnTe}$. b Mean grain

enable simultaneous evaporation in addition to a compositional control of $\mathrm{PbTe}$. The compounds phase-separate at the hetero-interface without forming an intermediate phase such as ternary solid solution $\mathrm{Zn}_{1-x} \mathrm{~Pb}_{x} \mathrm{Te}$. Also, a faceted $\mathrm{PbTe}$ nanocrystal forms polyhedron shape in ZnTe matrix. The ZnTe tends to grow [111] direction at the hetero-interface. The nanocomposite crystallizes even at $274 \mathrm{~K}$ (i.e., enabling a preparation at room temperature without postannealing), exhibiting temperature stability at relatively low substrate temperature. Current-voltage curve suggests that the ZnTe thin film prepared by the HWD apparatus exhibits n-type conductivity. Doping impurity will be needed for preparing $\mathrm{p}-\mathrm{n}$ junction using lead and zinc chalcogenide system.

Acknowledgments The present work was supported by a Grant-inAid for Scientific Research from the Japan Society for the Promotion of Science (No. 24360295). The author gratefully acknowledges the valuable comments of Grand-Fellow T. Masumoto [Research Institute for Electromagnetic Materials (DENJIKEN), Sendai, Japan]. The author is also grateful to Mr. N. Hoshi (DENJIKEN) and Ms. S. Sato (DENJIKEN) for assisting in the experiments.

Open Access This article is distributed under the terms of the Creative Commons Attribution 4.0 International License (http:// creativecommons.org/licenses/by/4.0/), which permits unrestricted use, distribution, and reproduction in any medium, provided you give appropriate credit to the original author(s) and the source, provide a link to the Creative Commons license, and indicate if changes were made.

\section{References}

1. Nozik, A.J.: Quantum dot solar cells. Phys E 14, 115-120 (2002) size of $\mathrm{PbTe}$ estimated from the diffraction peak of $\mathrm{PbTe}(\mathbf{a})$ as a function of the substrate temperature

2. Liu, D., Kamat, P.V.: Photoelectrochemical behavior of thin CdSe and coupled $\mathrm{TiO} / \mathrm{CdSe}$ semi-conductor films. J Phys Chem 97, 10769-10773 (1993)

3. Weller, H.: Quantum sized semiconductor particles in solution in modified layers. Ber Bunsenges Phys Chem 95, 1361-1365 (1991)

4. Zhu, G., Su, F., Lv, T., Pan, L., Sun, Z.: Au nanoparticles as interfacial layer for $\mathrm{CdS}$ quantum dot-sensitized solar cells. Nanoscale Res Lett 5, 1749-1754 (2010)

5. Hoyer, P., Könenkamp, R.: Photoconduction in porus TiO2 sensitized by PbS quantum dots. Appl Phys Lett 66, 349-351 (1995)

6. Chatterjee, S., Goyal, A., Shah, I.: Inorganic nanocomposites for next generation photovoltaics. Mater Lett 60, 3541-3543 (2006)

7. Yang, W., Wan, F., Chen, S., Jiang, C.: Hydrothermal growth and application of $\mathrm{ZnO}$ nanowire films with $\mathrm{ZnO}$ and $\mathrm{TiO} 2$ buffer layers in dye-sensitized solar cells. Nanoscale Res Lett 4, 1486-1492 (2009)

8. Abe, S., Ohnuma, M., Ping, D.H., Ohnuma, S.: Anatase-dominant matrix in $\mathrm{Ge} / \mathrm{TiO} 2$ thin films prepared by RF sputtering method. Appl Phys Exp 1, 095001 (2008)

9. Abe, $\mathrm{S}$.: InSb-added $\mathrm{TiO} 2$ nanocomposite films by RF sputtering. Nanoscale Res Lett 8, 269 (2013)

10. Lopez-Otero, A.: Hot wall epitaxy. Thin Solid Films 49, 3-57 (1978)

11. Abe, S.: One-step synthesis of $\mathrm{PbSe}-\mathrm{ZnSe}$ composite thin film. Nanoscale Res Lett 6, 324 (2011)

12. Abe, S.: One-step synthesis of composite thin film with $\mathrm{ZnSe}$ and $\mathrm{PbTeSe}$ ternary solid solution. Mater Renew Sustain Energy 4, 18 (2015)

13. Oba, Y., Abe, S., Ohnuma, M., Sato, N., Sugiyama, M.: Temperature dependence of nanostruc-ture in $\mathrm{PbSe}-\mathrm{ZnSe}$ composite thin film. J Phys D Appl Phys 47, 435102 (2014)

14. Title, R.S., Mandel, G., Morehead, F.F.: Self-compensation-limited conductivity in binary semiconductors. Phys Rev 136, A300 (1964)

15. Grytsiv, V.I., Tomashik, V.N., Olejnik, G.C., Tomashik, Z.F.: Investigation of the ZnTe-PbTe system [in Russian]. Izv AN SSSR Neorg Mater 16, 543-544 (1980)

16. Wise, F.W.: Lead salts quantum dots the limit of strong confinement. Acc Chem Res 33, 773-780 (2000) 
17. Nahory, R.E., Fan, H.Y.: Optical properties of zinc telluride. Phys Rev 825, 156 (1967)

18. Zemel, J.N., Jensen, J.D., Schoolar, R.B.: Electrical and optical properties of epitaxial films of $\mathrm{PbS}, \mathrm{PbSe}, \mathrm{PbTe}$, and $\mathrm{SnTe}$. Phys Rev 140, A330-A342 (1965)

19. Rawat, P.R., Paul, B., Banerji, P.: Exploration of Zn resonanceand thermoelectric properties in I-doped $\mathrm{PbTe}$ with $\mathrm{ZnTe}$ nanostructures. ACS Appl Mater Interfaces 6, 3995-4004 (2014)

20. Pashinkin, A.S., Novoselova, A.V.: Opredelenie davleniya nasyshchennogo para tverdogo telluridasvintsa [in Russian]. Zhur Neorg Khim 4, 2657-2660 (1959)

21. Korneeva, I., Belyaev, A.V., Novoselova, A.V.: Determing saturated vapor pressure of solid zinc andcadmium tellurides [in Russian]. Zhur Neorg Khim 1, 5-12 (1960)

22. Brebrick, R.F., Straus, A.J.: Partial pressures in equilibrium with group IV tellurides. I. Optical ab-sorption method and results for PbTe. J Chem Phys 40, 3230-3241 (1964)
23. Goldfinger, P., Jeunehomme, N.: Mass spectrometric and Knudsen-cell vaporization studies of group 2B-6B compounds. Trans Fraday Soc 59, 2851-2867 (1963)

24. Liu, N.Z., Li, G.H., Zhu, Z.M., Han, H.X., Wang, Z.P., Ge, W.K., Sou, I.K.: Raman scattering and photoluminescence of $\mathrm{ZnS}_{x} \mathrm{Te}_{1-x}$ mixed crystals. J Phys Condens Matter 10, 4119-4129 (1998)

25. Koguchi, N., Kiyosawa, T., Takahashi, S.: Double hetero structure of $\mathrm{Pb}_{1-x} \mathrm{Cd}_{x} \mathrm{~S}_{1-y} \mathrm{Se}_{y}$ lasers grown by molecular beam epitaxy. J Cryst Growth 81, 400-404 (1987)

26. Wang, Z.L.: Transmission electron microscopy of shape-controlled nanocrystals and their assemblies. J Phys Chem 104, 1153-1175 (2000)

27. Guinier, A.: X-ray diffraction in crystals, imperfect crystals, and amorphous bodies. Dover Publications, Mineola (1994)

28. Brus, L.E.: A simple model for the ionization potential, alaectron affinity, and aqueous redox potentials of small semiconductor crystallites. J Chem Phys 79, 5566-5571 (1983) 